\title{
LATERAL AND LONGITUDINAL CONTROL SYSTEM FOR THE AUTOMATION OF AN ARTICULATED BUS
}

\author{
H. MONTES ${ }^{1,2}$, C. SALINAS ${ }^{1}$, J. SARRIA ${ }^{1}$ and M. ARMADA ${ }^{1}$ \\ ${ }^{1}$ Centre for Automation and Robotics, CSIC, Spain ${ }^{2}$ Faculty of Electrical Engineering, \\ University Technological of Panama, Panama \\ hmontes@iai.csic.es
}

\begin{abstract}
This paper presents several results regarding the lateral and longitudinal control systems that have been applied for the automation of an articulated bus, using a rolling wheeled box system with special design that moves inside a guide rail. Nowadays, transport systems are achieving major advances by the incorporation of automation based technologies. Recent developments of electronic instrumentation and actuation systems and the increasing speed of processors allows for the implementation of real-time systems. The automation of an articulated bus provides combined advantages of both conventional bus and train, because it can ascend slopes of $15 \%$ and turn on curves of low radius. This transport modality is an interesting, low cost and friendly option. In this paper an experimental setup for the development of lateral and longitudinal control of the articulated bus is presented. Comprised by an experimental mobile platform (articulated bus) fully instrumented and a ground test area of asphalt roads inside CSIC installations, this experimental facility allows full testing of automatic driving systems.
\end{abstract}

Keywords: Transport Systems, Bus Rapid Transit, autonomous transport systems, lateral and longitudinal control, perception.

\section{Introduction}

The development of automated vehicles has been the subject of important research activities, where control systems play a very relevant role [1]. By other side, the interest of new, safer, more reliable, mass transportation systems is of growing interest, leading to Intelligent Transport Systems (ITS) [2-3]. Control of vehicle dynamics has been increasingly investigated over the last 15-20 years [4-8]. In addition, several lateral and longitudinal control designs are documented in the literature [9-11]. Bus Rapid Transit systems are a promising transportation system that is becoming very popular and their automation is of major relevance [2].

In this paper an experimental platform for research on automatic control of articulated bus is presented. The aim of the platform is to allow full 
experimentation in real conditions for testing technology developments and control algorithms. Platform consists in an articulated bus, Volvo BM10, fully instrumented and in a ground test area composed of asphalt roads inside CSIC premises [9].

In addition, some experimental results of lateral and longitudinal control for the automation of the articulated bus are presented, when the vehicle follow the test lane.

\section{Description of the system}

The experimental platform purpose is to provide a reliable starting point to carry out research on automatic control of articulated buses. Figure 1 shows the main elements of the overall architecture of the experimental platform set-up using a Volvo B10M articulated bus. This experimental platform is used on a private inner road facility located at CSIC premises. There are a number of applied research projects involving several Spanish Industrial Companies engaged in transport issues that are being realized in co-operation with Centre for Automation and Robotics of the CSIC.

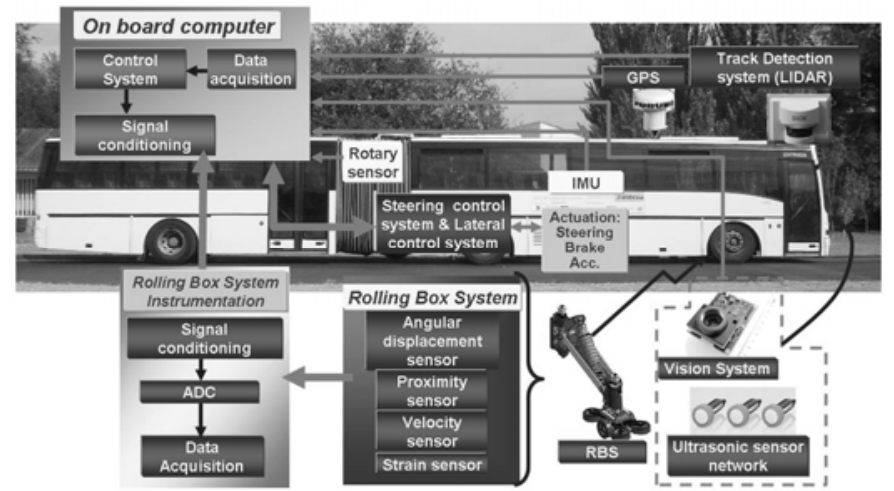

Figure 1. Architecture of the systems of perception and actuation for automation of articulated bus.

This paper presents the developments regarding lateral and longitudinal control of the articulated bus, using the steel box on wheels with special design (RBS) installed on the axle of the front wheels of the vehicle. Figure 2 shows the scheme of the general control system applied in the articulated bus.

The control system consists of two types of guide rail detection, the first is through the RBS, which detects the guide rail to about $0.5 \mathrm{~m}$ ahead of the axle of the front wheels of the bus, and the second is through the vision artificial system, consisting of a LIDAR, which provide the position in advance of the 
test lane to the control system. This position is variable and can be adjusted between 3-8 meters [12]. In this paper only describes the lateral and longitudinal control of the bus, without the make use of LIDAR. However, the data in advance of the test lane, provided by the artificial vision system, will be added to the lateral and longitudinal control system for improving the test lane tracking.

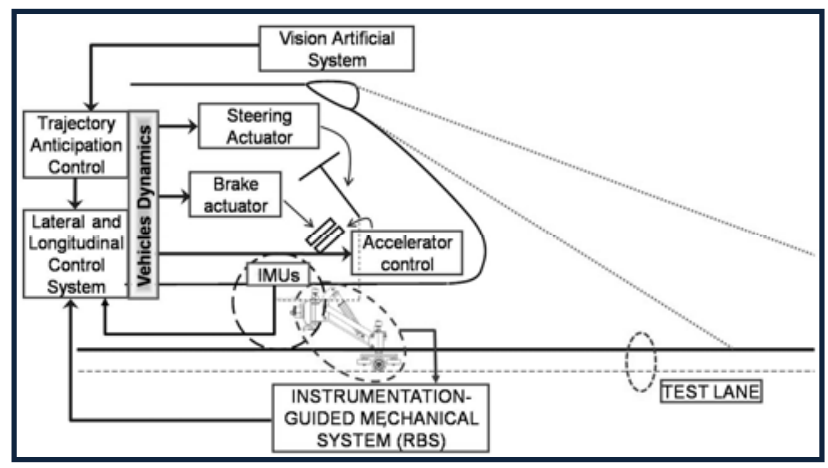

Figure 2. General control system scheme.

\section{Description of the lateral and longitudinal control}

The longitudinal control of the articulated bus Volvo BM10 is carried out so that the vehicle follow the test lane at a speed previously set. This speed is relatively low due to the sinuosity of the trajectory. The average speed for most of the path is $2 \mathrm{~m} / \mathrm{s}$. However, in the straight section of the path, the speed goes up to $6 \mathrm{~m} / \mathrm{s}$.

Figure 3 shows a longitudinal control scheme implemented in the bus. The desired speed is relatively low so that the bus can follow the track properly. The control system acts on the PotBox of the bus, which it has an action range of 0 to 5 DC volts, rather than acting on the throttle. The control over acceleration/speed of the bus is better in this case. The acceleration is limited in order to protect the PotBox and not to exceed the desired speed over limits that cannot be controlled.

When the bus speed exceeds the desired speed, then the acceleration controller stops and selects the brake controller. The brake controller has minimum and maximum limits, which determines the workspace for this controller. Within these limits, the controller generates a sawtooth signal to act over the set driver and brake actuator, which consists of a DC servomotor. This makes the brake subsystem perform a bus stop in a gentle decelerate. When the 
speed decreases to a preset threshold, the brake subsystem stops acting, and starts the accelerator subsystem. The bus speed is measured by a speed sensor installed on the RBS, whose resolution is greater than that offered by the bus.

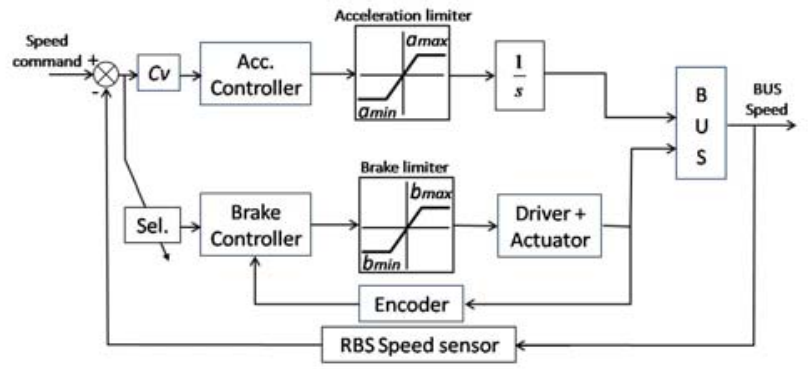

Figure 3. Longitudinal control scheme.

On the other hand, an electric actuator composed of a set DC motor, gearbox and encoder has been installed on the steering column. The actuator receives commands from the lateral control system, so that the vehicle follows the monorail during implementation of tasks.

Figure 4 shows the lateral control scheme, implemented in the bus. It takes into account the steering limit, which in this case is $\pm 900^{\circ}$. At the same time makes the axle of the front wheel moves between $\pm 45^{\circ}$.

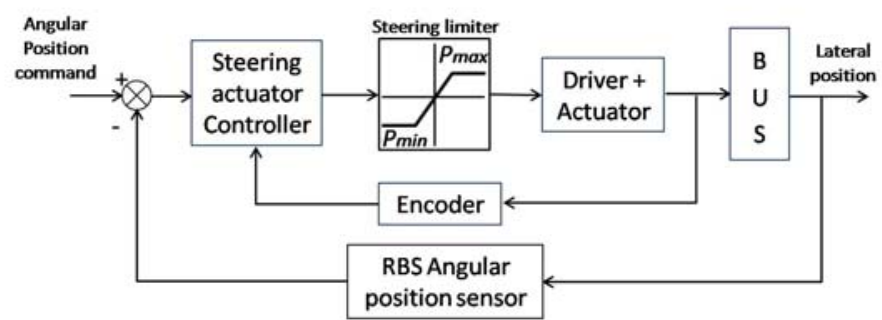

Figure 4. Lateral control scheme.

The feedback of the lateral control is performed by mean of angular position sensors located on the guided mechanical system. This system has two dof, one located on the front axle of the bus (top dof) and the other on the box with wheels located inside of the rail (bottom dof), on the horizontal plane of the bus.

The sum of the displacement of two dof provides the position of the lane. However, the control system observes in detail the top dof, which should not exceed $\pm 45^{\circ}$ with respect to the longitudinal axis, to avoid straining the torsion 
springs that are located there. In addition, it limits the lateral movement of the bus, to avoid derailment or unwanted obstructions of the system. The length of the mechanical system of guidance $(0.5 \mathrm{~m})$ and placement on the front axle of the bus makes experimental tests are conducted at low speed.

\section{Experimental results}

To evaluate different sensing and actuation systems for automatic control of articulated bus (implementing strategies of lateral and longitudinal control), the mobile platform is complemented with a ground facility, where infrastructure modifications can be made to investigate new transport systems [13]. Figure 5 shows the top and panoramic view of the test lane, which is $385 \mathrm{~m}$ long.

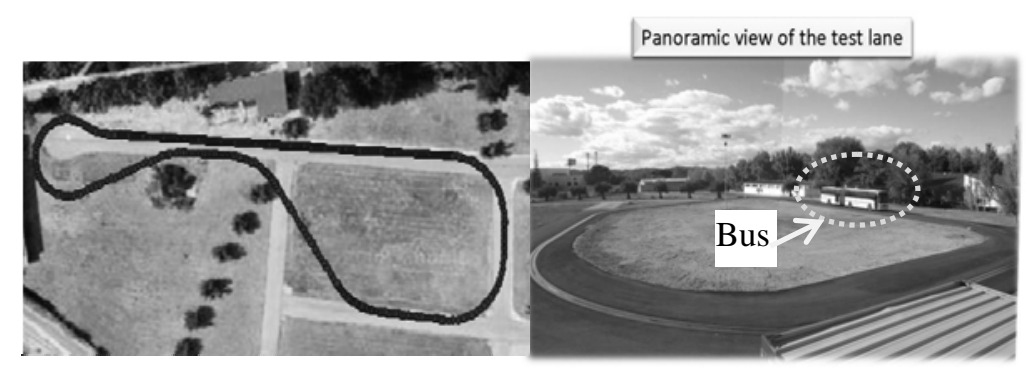

Figure 5. Panoramic views of the new monorail test lane.

The results of longitudinal control can be seen in Figure 6. This Figure 6 compares the speed of the vehicle, the position of the brake and acceleration in the longitudinal axis obtained through an IMU440CA installed on the front axle.

During most of the trajectory, the commanded speed was $2 \mathrm{~m} / \mathrm{s}$, because the test lane contains small radii of curvature, and slope with considerable inclinations. In last trajectory segment of the test lane (straight line), the speed was increased up to $6 \mathrm{~m} / \mathrm{s}$. The application of the brake is to support the speed commanded during the slope trajectory. This takes place between the times $125 \mathrm{~s}$ and 300s, approximately. The oscillatory signal produces a comfortable sensation of deceleration. 


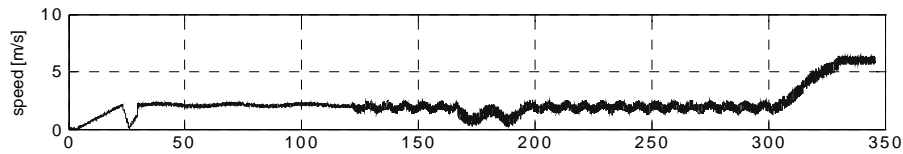

[s]
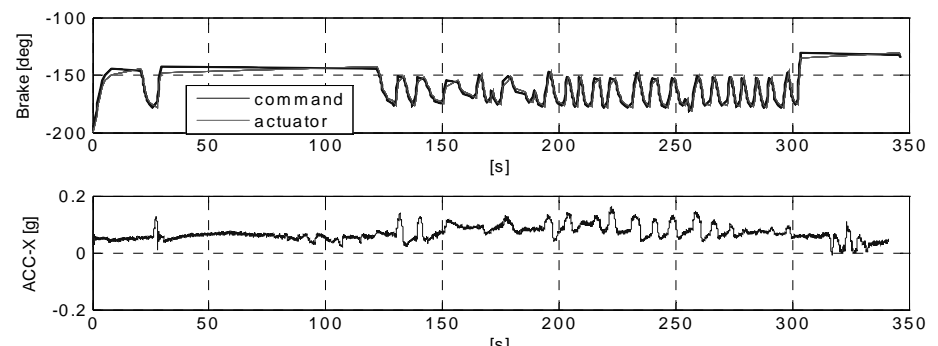

Figure 6. Comparison among bus speed, brake position and $\mathrm{x}$-acceleration during the tracking trajectory.

Figure 7 shows the experimental results obtained by applying the lateral control of the bus for tracking monorail. This figure shows the steering command, the lateral acceleration of the bus, and angular rate on $\mathrm{Z}$ axis. The angular rate sensor follows the curves of the trajectory commanded by the bus steering. The lateral acceleration signal will be used in a feedback loop lateral control to improve the tracking of the trajectory. Using this signal in the control system, small lateral movements will be diminished.
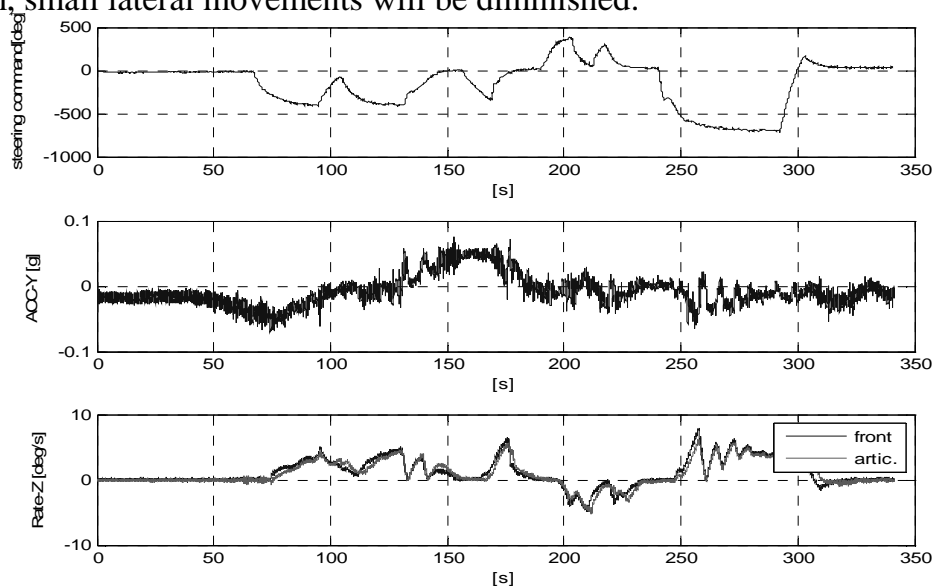

Figure 7. Comparison among steering command, lateral acceleration and angular rate in $\mathrm{z}$ axis during the tracking trajectory.

The lateral error during the tracking of test lane is relatively small. Figure 8 shows that the maximum lateral error was approximately $0.35 \mathrm{~m}$ in an 
experimental test. This makes the maximum angular displacement with respect to the longitudinal axis of the bus to be $44^{\circ}$. This value is within the safety margins established in the control strategy.

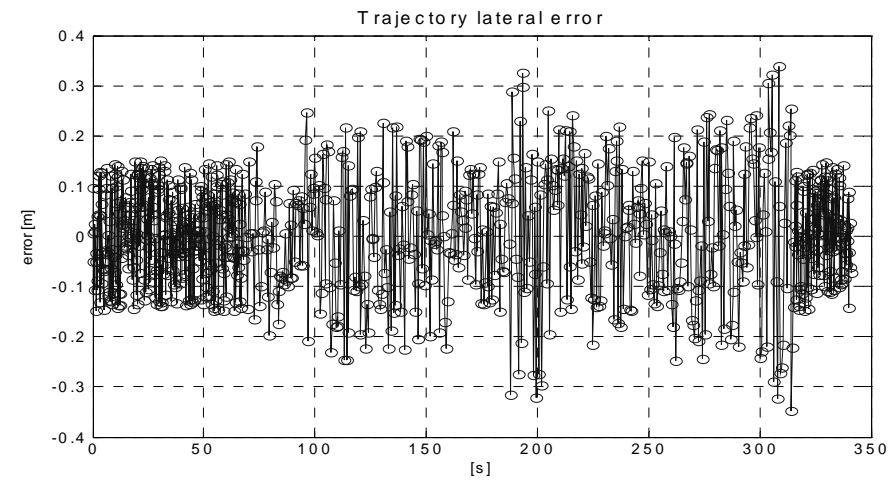

Figure 8. Lateral error during the tracking of test lane.

Figure 9 shows an experimental result of the lateral and longitudinal control applied on the articulated bus Volvo BM10. The trajectory in Figure 9 only represents a sector of the test lane. It is possible to see the monorail and the steering wheel in several positions.

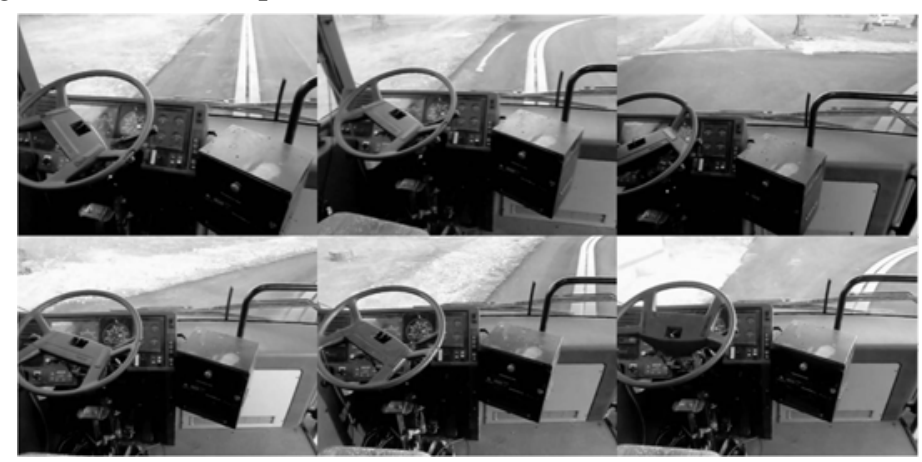

Figure 9. Details of the wheel and the track during one of the experimental tests.

\section{Conclusions}

In this paper an experimental platform for research on automatic control of articulated bus has been presented. Apart from the mobile system (a Volvo B10M) fully instrumented, a ground infrastructure was also introduced.

Experimental results regarding lateral and longitudinal control of articulated bus were also offered. The lateral control will be improved with the introduction 
of Kalman Filter in the control algorithm. Moreover, excellent results have been presented for the detection of the guide rail through of the steel box instrumented with several sensors.

\section{Acknowledgements}

The authors acknowledge partial funding of this research under: Robocity2030 S-2009/DPI-1559, and IMADE PIE/62/2008 (Comunidad de Madrid, ALDESA CONSTRUCCIONES SA, MAXIMASDE); FORTUNA D/026706/09 (Agencia Española de Cooperación Internacional para el Desarrollo, AECID). Dr. Héctor Montes acknowledges support form Universidad Tecnológica de Panamá and from CSIC under grant JAE-Doc.

\section{References}

1. R. Fenton, G. Melocik and K. Olson, IEEE Transactions on Automatic Control, 21(3), 306 (1976).

2. S. Shladover, C. Desoer, J. Hedrick, M. Tomizuka, J. Walrand, W. Zhang, D. McMahon, H. Deng, S. Sheikholeslam and N. McKeown, IEEE Transaction on Vehicular Technology, 40(1), 114 (1991).

3. W. C. Collier and R. J. Weiland, IEEE SPECTRUM, 31(4), 27 (1994).

4. J. Ackermann, IEEE Control Systems Magazine, 23 (1997).

5. U. Kiencke and A. Daiß, Control Eng. Practice, 5(8), 1145 (1997).

6. J.C. Gerdes and E.J. Rossetter, Proc. ASME IMECE, Nashville, TN. (1999).

7. S. Mammar, S. Glaser and M. Netto, Proc. American Control Conference, 14 (2006).

8. P. Falcone, F. Borrelli, J. Asgari, H. Tseng and D. Hrovat, IEEE Trans. Contr. Syst. Tech., 15(3), 566 (2007).

9. J. Ackermann and W. Siegel, Proc. American Control conference, 795 (1990).

10. J. Ackermann, J. Guldner, W. Siegel, R. Steinhauser and V.I. Utkin, Proc. IEEE Transactions on Control System Technology, 3(1), (1995).

11. R.H. Byrne, C.T.Abdallah and P. Dorato, IEEE Control Systems, 18(2), 70 (1998).

12. C. Salinas, M. Armada and H. Montes, Proc. of CLAWAR2010, Nagoya, Japan (2010).

13. H. Montes, C. Salinas, J. Sarria and M. Armada, Proc. IARP Workshop on Service Robotics and Nanorobotics, (2009). 